\title{
Compact Groove Diamond Antenna in Gap Waveguide Technology with Broadband Circular Polarization at Millimeter Waves
}

\author{
Dayan Pérez-Quintana, Alicia Torres-García, Iñigo Ederra and Miguel Beruete
}

\begin{abstract}
In this paper, three compact antennas using the Ridge Gap Waveguide (RGW) technology working in the millimeter-wave band $(60 \mathrm{GHz})$, with a high-purity and broadband circular polarization (CP) are numerically and experimentally analyzed. The structure is fed from the bottom by means of a standard WR-15 waveguide (V-band) to make it compatible with standard measurement systems. It is coupled with a miniaturized step transition to a ridgeline that ends in two arms of different lengths. $\mathrm{CP}$ is generated in a simple and effective way, by means of two orthogonal feeder arms that excite a CP in a diamond-shaped slot on top. Simulations and measurements have an excellent agreement reaching a matching bandwidth $\left(\mathrm{S}_{11}<\right.$ $-10 \mathrm{~dB}$ ) from 60.3 to $69.6 \mathrm{GHz}(>9 \mathrm{GHz})$. Applying the axial ratio criterion $(A R<3 \mathrm{~dB})$ the bandwidth in $\mathrm{CP}$ is $14.48 \%$, with respect to the central frequency $(59$ to $70 \mathrm{GHz}$ ). The maximum gain is obtained with the most evolved design incorporating a diamond aperture with a horn taper and a circular groove, reaching a value of $11.12 \mathrm{~dB}$ at $67.3 \mathrm{GHz}$.
\end{abstract}

Index Terms - millimeter-waves, gap waveguide technology, circular polarization, planar antenna.

\section{INTRODUCTION}

$\mathrm{T}$ THE gradual saturation of the lower part of the radio spectrum has spurred research towards efficient antennas and feeding methods in higher bands, mainly millimeter and terahertz waves. Nevertheless, a fundamental obstacle that must be circumvented to achieve a full development of high frequency technology is the problem of guiding waves with low loss. Conventional dielectric materials have increasing loss as frequency approaches the millimeterwaves band, a fact that rules out planar solutions such as microstrip and coplanar waveguides [1], [2]. The loss of conductors also rises with frequency mainly due to the roughness introduced by the conventional manufacturing techniques, which is no longer negligible at millimeter wavelengths. In addition, standard metallic waveguides require

This work was supported by the Ministerio de Ciencia, Innovación y Universidades under Grant RTI2018-094475-B-I00 (MCIU/AEI/FEDER, UE). (Corresponding author: Miguel Beruete)

D. Pérez-Quintana, is with the Antennas Group, Universidad Pública de Navarra, 31006 Pamplona, Spain, and also with the Institute of Smart Cities (ISC), Universidad Pública de Navarra, 31006 Pamplona, Spain (e-mail: dayan.perez@unavarra.es)

A. Torres-García is with the Antennas Group, Universidad Pública de Navarra, 31006 Pamplona, Spain, and also with the Institute of Smart Cities precise screwing or welding techniques when they are fabricated in two blocks, introducing irregularities in the surface that deter further the performance of the guide.

An attractive alternative to overcome these limitations is the use of gap waveguide (GW) technology [3], [4]. GW shows considerable advantages at millimeter waves such as low loss (most of the designs are fully metallic), no need of electric contact and adaptability to plane surfaces, given its simple design. They also have a lower manufacturing cost with respect to traditional hollow waveguides, since the tolerances are coarser alleviating the fabrication constraints.

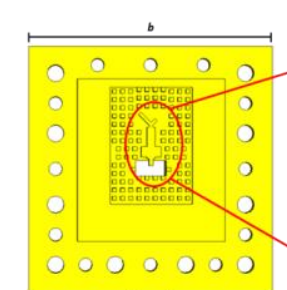

(a)

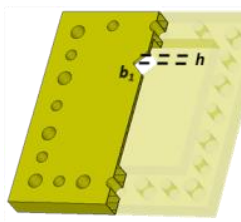

(d)

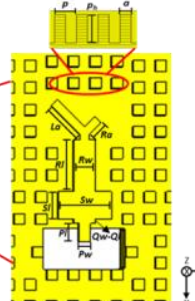

(b)

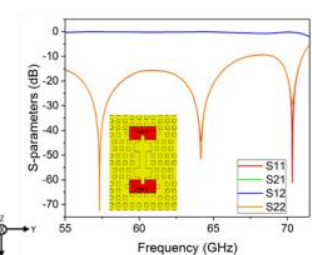

(c)

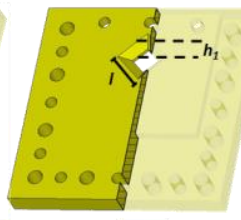

(e)

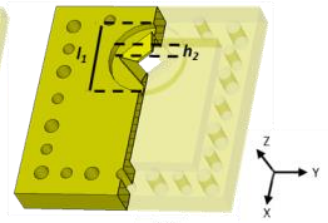

Fig 1. (a) Schematic of the antennas feeding, top view. (b) Detailed view of the antenna feeding system and main parameters. (c) Simulated reflection and transmission coefficients of feeding system using a back to back evaluation. (d) D antenna top view. (e) DH antenna top view. (f) DHG antenna top view.

During the last decade, GW technology has developed three principal variants: groove-gap waveguide (GGW) [4], ridgegap waveguide (RGW) [5]-[7] and microstrip-gap waveguide (MGW) [8]. As the latter has generally higher loss due to the need of a dielectric slab, the other technologies, which are fully metallic, are preferred at millimeter-waves and terahertz

(ISC), Universidad Pública de Navarra, 31006 Pamplona, Spain (e-mail: aliciaelena.torres@unavarra.es).

I. Ederra is with the Antennas Group, Universidad Pública de Navarra, 31006 Pamplona, Spain, and also with the Institute of Smart Cities (ISC), Universidad Pública de Navarra, 31006 Pamplona, Spain (e-mail: inigo.ederra@unavarra.es).

M. Beruete is with the Antennas Group-TERALAB, Universidad Pública de Navarra, 31006 Pamplona, Spain, and also with the Institute of Smart Cities (ISC), Universidad Pública de Navarra, 31006 Pamplona, Spain (e-mail: miguel.beruete@unavarra.es). 


\section{frequencies.}

$\mathrm{RGW}$, the solution implemented in this paper, is based on the use of two parallel plates, a perfect electrical conductor (PEC) and a perfect magnetic conductor (PMC), as well as a central ridge, that can also be modelled as PEC [9], [10]. The PMC provides a high impedance surface opening a forbidden band around the frequency of resonance that prevents the propagation of the electromagnetic waves confining them in a prescribed region. The wave is then effectively guided between the ridge and the PEC plate. A PEC medium can be easily approximated with good electrical conductors. However, obtaining a PMC plate is not that simple, as this type of material does not exist naturally. Therefore it must be emulated by Artificial Magnetic Conductors (AMC) [11] such as mushroom-like surfaces or a bed of nails, which is the solution implemented in this paper [12].

Circular polarization (CP) in wireless communications systems has several advantages over linear polarization: $\mathrm{CP}$ does not require polarization alignment between the transmitter and the receiver and is more robust against multipath effects [13]. To date, there are several examples of CP antennas based on GW technology. In [14], a CP antenna operating in the band $75-80 \mathrm{GHz}$ was demonstrated by using rotated rectangle slot arrays on a bed of nails. It used a RGW scheme to feed an array of $1 \times 4$ elements. Following a similar procedure a $\mathrm{RGW}$ antenna based on slot arrays operating at the Ka-band was presented in [15], [16]. In [15], CP was generated by means of an array of $2 \times 2$ annular slot elements, implementing a power divider directly in RGW technology. An interesting evolution of $\mathrm{GW}$ techniques was presented in [16] by combining RGW and GGW in an antenna array of $4 \times 4$ chamfered cylindrical radiating apertures fed by a corporate GW network. Another way to design $\mathrm{CP}$ antennas using MGW was demonstrated in [17]. There, the antenna was composed of two substrates, with a ring slot etched on the upper substrate, able to generate CP. This resulted in a compact structure, with MGW technology to feed the radiant element.

In this paper, we design and analyze three compact $\mathrm{CP}$ antennas using the RGW technology. $\mathrm{CP}$ is generated in a simple and effective way, by means of two orthogonal feeder arms that excite a CP in a diamond-shaped slot on top [18]. The basic design is then evolved gradually by introducing first a taper in the diamond-slot and then inserting it in a circular groove, achieving a notable increase in the gain without deterring the other radiation parameters. An impedance BW of approximately $9 \mathrm{GHz}$ is achieved, which represents $14.48 \%$ of the center frequency. The gain of the different prototypes goes between 5.49 and $11.12 \mathrm{~dB}$, which are relatively high values considering that the antenna has a single element. Excellent polarization purity with an AR below $2 \mathrm{~dB}$ within a BW of more than $10 \mathrm{GHz}(15.75 \%)$ is demonstrated. All antennas have right-handed $\mathrm{CP}$ (RHCP) with polarization discrimination of more than $15 \mathrm{~dB}$. The prototypes devised show that it is possible to design and manufacture $\mathrm{CP}$ antennas with large operation BW and moderate gain in GW technology in a novel, simple and very compact technique, without the need to implement arrays and complex feeding networks.

\section{Design AND Simulation ReSUlts}

All the antennas in this paper were designed and optimized to operate in the V-band of millimeter-waves, specifically from 55 to $70 \mathrm{GHz}$, using the Transient Solver of the commercial simulator CST Microwave Studio®. The bottom plate containing the feeding network is the same for all the designed antennas and is based on RGW technology, as shown in the general view of Figs. 1(a) and (b). The structure is fed from the bottom by means of a standard WR-15 waveguide to make it compatible with standard measurement systems based on vector network analyzers, see details in Fig 1(b) and inset of Fig. 1(c).

Three different upper plates were implemented, each one of them corresponding to different evolution stages of the antenna. The basic design is a diamond-shaped (D) slot antenna put on top of the feeding network, see Fig. 1(d). The first evolution from this initial prototype consists in introducing a taper in the upper face, following a strategy similar to horn antennas but in a much more compact profile, leading to the diamond-horn (DH) antenna depicted in Fig. 1(e). In the last evolution step, a circular groove concentric to the DH slot is carved on the top plate to increase the gain. This last design, shown in Fig. 1(f), is called diamond-horn-groove (DHG) antenna. The dimensions of all prototypes are summarized in Table I. The material employed in all the structures is aluminum, due to its good conductivity in the operation band $\left(\sigma_{\mathrm{Al}}=3.72 \times 10^{6} \mathrm{~S} / \mathrm{m}\right)$, mechanical robustness and compatibility with standard manufacturing techniques.

\begin{tabular}{ccc}
\multicolumn{3}{c}{ TABLE I } \\
\hline \hline Parameter & Description & \\
\hline$b$ & Antenna length & Values $\left(\mathrm{mm} / \lambda_{0}{ }^{*}\right)$ \\
$b_{l}$ & Diamond length & $30 / 6.36$ \\
$h$ & Diamond thickness & $2.65 / 0.56$ \\
$l$ & Horn slot length & $0.5 / 0.10$ \\
$h_{l}$ & Horn thickness & $5.08 / 1.07$ \\
$l_{l}$ & Groove diameter & $1.45 / 0.31$ \\
$h_{2}$ & Groove thickness & $10.93 / 2.31$ \\
$l_{a}$ & Left arm length & $1.28 / 0.27$ \\
$r_{a}$ & Right arm length & $1.77 / 0.38$ \\
$r_{l}$ & Ridge length & $0.64 / 0.14$ \\
$r_{w}$ & Ridge width & $2.17 / 0.46$ \\
$p_{w}$ & First step width & $1.0 / 0.21$ \\
$p_{l}$ & First step length & $0.54 / 0.11$ \\
$q_{w}$ & Second step width & $0.83 / 0.18$ \\
$q_{l}$ & Second step length & $0.23 / 0.048$ \\
$s_{w}$ & Third step width & $0.2 / 0.04$ \\
$s_{l}$ & Third step length & $2.45 / 0.52$ \\
$a$ & Pin width & $1.05 / 0.22$ \\
$p$ & Pin period & $0.5 / 0.10$ \\
$p_{h}$ & Pin height & $1.0 / 0.21$ \\
$g$ & Air gap & $1.5 / 0.32$ \\
${ }^{*} \lambda_{0}=4.72$ at $63.5 \mathrm{GHz}$ & $0.2 / 0.04$ \\
& &
\end{tabular}

As commented above, standard experimental setups are based on standard rectangular waveguides, so the first step is to design and optimize a WR-15/RGW transition [5], [19]-[23]. This is a critical aspect, since our main goal is to achieve a broad operation $\mathrm{BW}$ with $\mathrm{CP}$ operation while maintaining a low profile structure and, therefore, this transition should not degrade the overall performance. To implement it, we take inspiration from [5] wherein a similar transition was used in the $\mathrm{Ku}$-band. Our design working in the V-band is shown in Fig. 
1(b). It is a stepped impedance matching network composed of three subsections: the first part is the narrowest, with width labelled as $p_{w}$; it serves to provide coupling from the fundamental WR-15 waveguide mode and the fundamental RGW mode. The second step is the shortest of all with dimensions $q_{w} \times q_{l}$ (Table I), and acts as a smooth stepped transition. The third stage is a wide and long block of dimensions $s_{w} \times s_{l}$ responsible for the final coupling of the signal and delivery to the ridge $\left(r_{w}\right)$, which feeds upper aperture. As shown in Fig. 1(c) the transition has a good matching within the considered band, with a reflection coefficient below $-10 \mathrm{~dB}$ and negligible insertion loss. These curves were obtained by placing two ports on both sides on the bottom WR-15 waveguide, making a back to back evaluation and analyzing the ridge-line behavior, as depicted in the inset of Fig. 1(c).

$0^{\circ}$

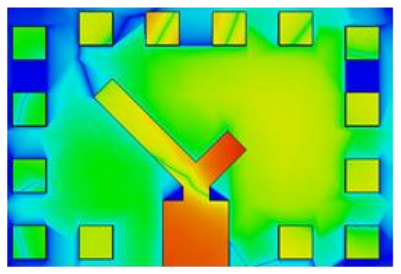

$180^{\circ}$

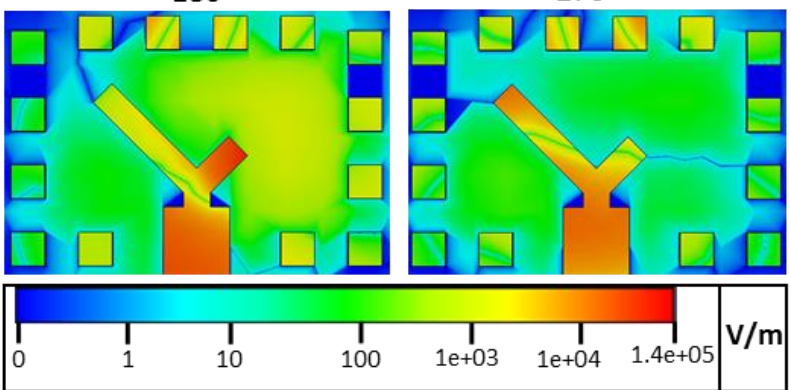

Fig. 2. Electric field magnitude at $63.5 \mathrm{GHz}$ at four different snapshots, showing that the ending arms are operating in quadrature.

To generate the $\mathrm{CP}$, the feeding ridge is finished in two orthogonal arms of different lengths. From the dimensions shown in Table I, it can be observed that the length difference of the arms is $1.13 \mathrm{~mm}$, which corresponds approximately to $\lambda_{0} / 4$, where $\lambda_{0}=4.72 \mathrm{~mm}$ is the wavelength at the operation frequency, $f_{0}=63.5 \mathrm{GHz}$. This guarantees that the phase difference between both arms is close to $90^{\circ}$, as demonstrated in Fig. 2, which shows that the electrical field distribution in both arms is in quadrature: the maximum value in one arm corresponds to a minimum in the other.

With this feeding system, the surface current excited on the upper plate has a clear spiral pattern, as shown in Fig. 3 (particularized to the basic D antenna), as corresponds to a CP. As the phase increases, the currents rotate, indicating that the antenna supports right-handed CP (RHCP). It is noteworthy that with such a simple feeding system it is possible to generate $\mathrm{CP}$ in a compact footprint, in contrast with the typical approach based on an array of antennas, which increases the complexity as well as the footprint area.
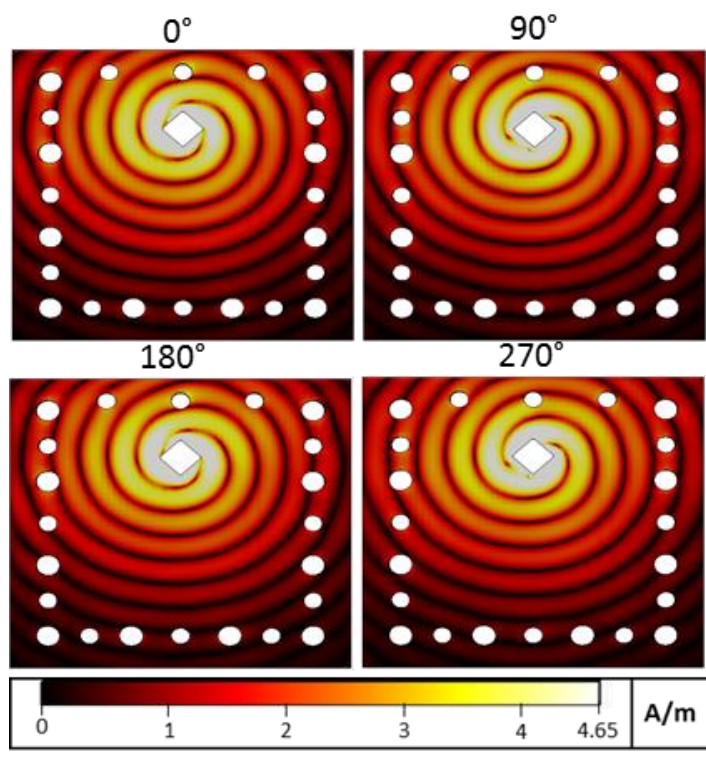

Fig. 3. Top view of the surface current magnitude on the top plate (D antenna) at $63.5 \mathrm{GHz}$ and four different snapshots.

Fig. 4 shows the simulation results for the three structures considered. Their performance is evaluated in terms of the reflection coefficient, axial ratio (AR), broadside gain and polarization purity. First, we note that all the antennas are matched from 59 to $69 \mathrm{GHz}$ approximately, with the criterion of having a reflection coefficient magnitude below $-10 \mathrm{~dB}$, see Fig. 4(a). The best case happens for the DHG antenna (blue curve), where a broad matching BW of $9.6 \mathrm{GHz}(15.12 \%)$ from 59.3 to $68.9 \mathrm{GHz}$ is achieved. The other antennas have a similar performance with a BW of $9.22 \mathrm{GHz}(14.51 \%)$ from 58.95 to $68.17 \mathrm{GHz}$, in the case of the $\mathrm{D}$ antenna and $9.28 \mathrm{GHz}$ (14.61\%) from 59.08 to $68.36 \mathrm{GHz}$ for $\mathrm{DH}$ antenna. In the reflection coefficient spectrum, one can notice two main resonances, one at 63.5 and another at $65.5 \mathrm{GHz}$ (D antenna red curve). These resonance frequencies cannot be ascribed to any isolated arm, demonstrating clearly that the mechanism is based on a close interaction between them. Interestingly, what one can identify are two orthogonal resonances in the slot, each one of them corresponding to the aforementioned frequencies. Finally, we think that beyond this qualitative description, the interpretation based on a length difference between arms of $\lambda_{0} / 4$ might be sufficient to explain the basic principle behind the circular polarization generation. Note that the minimum AR happens exactly in the middle of both resonance frequencies. Although these results correspond to the D antenna (which is the simplest one), a similar analysis holds for the other designs.

The evolution from the basic D antenna to the more complex DHG antenna was done in order to increase the gain of the design, keeping the same footprint to ensure a compact structure. The $\mathrm{D}$ prototype has a relatively low gain with a maximum around $7 \mathrm{~dB}$, as corresponds to a small slot antenna, see red curve in Fig. 4(b). By introducing a taper in the diamond slot the gain can be increased to $9 \mathrm{~dB}$, see the yellow curve in Fig. 4(b). This tapering is inspired by the operation principle of horn antennas, but in this case with a very low profile. To further increase the gain, a concentric circular groove was 
introduced around the DH slot. The groove operation can be interpreted as a cavity that boosts the radiation in the broadside direction. In addition, the difference between the groove radius and the edge of the inner diamond slot is approximately $\lambda_{0}$ so it interferes in phase with the slot at broadside, enhancing the gain in that direction. This latter design (blue curve) has a peak gain of more than $11 \mathrm{~dB}$ at $64.5 \mathrm{GHz}$ and in the whole operation band is above $10 \mathrm{~dB}$.

Another important figure of merit to evaluate the performance and delimit properly the operation $\mathrm{BW}$ of $\mathrm{CP}$ antennas is the AR. Fig. 4(c) shows the simulated AR curves for all the antennas considered. By analyzing the results, it can be concluded that all the designed antennas generate $\mathrm{CP}$ of high purity with low values of AR in the considered frequency span, especially the basic D antenna and the most evolved DHG antenna. The CP BW of the antennas is obtained by applying the criterion of having AR $<3 \mathrm{~dB}$. From the curves it is seen that the DH antenna achieves a BW of $9.94 \mathrm{GHz}$ (from 59 to $68.94 \mathrm{GHz}$ ), which means a fractional BW of $15.65 \%$. The D and DHG antennas have a BW of $10.7 \mathrm{GHz}$ (from 59.3 to $70 \mathrm{GHz}$ ), or equivalently a fractional $\mathrm{BW}$ of $16.6 \%$. It is remarkable the excellent performance of the DHG antenna, with $\mathrm{AR}<1.5 \mathrm{~dB}$ within a broad BW from 60.7 to $70 \mathrm{GHz}$. It is seen in that figure that the DHG antenna generates CP even beyond $70 \mathrm{GHz}$. However, the impedance matching is only good up to $68.9 \mathrm{GHz}$, restricting the practical operation BW to this frequency.
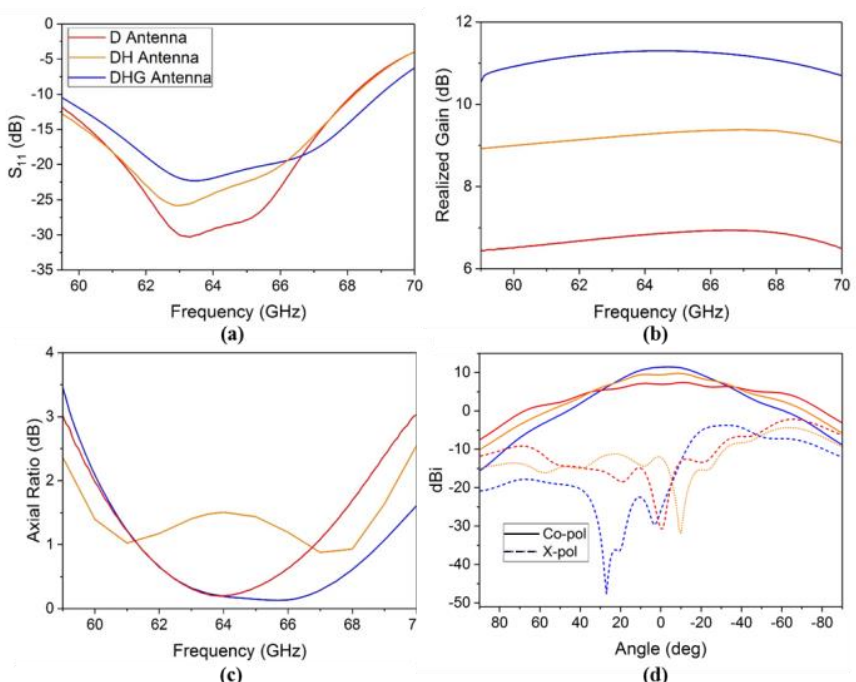

(c)

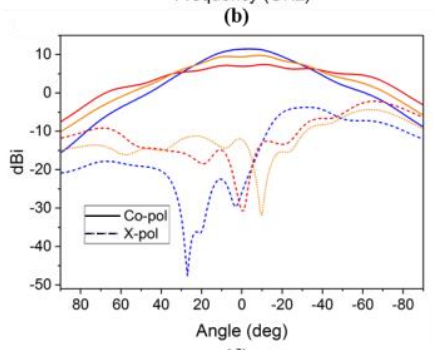

(d)

Fig. 4. Simulated results of the three designed antennas. (a) Reflection coefficient magnitude. (b) Axial ratio. (c) Realized gain at broadside $\left(\varphi=0^{\circ}\right.$ and $\left.\theta=0^{\circ}\right)(\mathrm{d})$ Co-polarized and cross-polarized radiation pattern at $63.5 \mathrm{GHz}$

Finally, Fig. 4(d) shows the radiation pattern of all the designs at $63.5 \mathrm{GHz}$ at broadside. Each curve analyzed has more than $20 \mathrm{~dB}$ of cross polarization isolation, showing that the antennas have an excellent polarization purity and then respond mainly to RHCP.

\section{MANUfACtURING AND EXPERIMENTAL RESUltS}

To corroborate experimentally the previous simulation results, both the D and DHG antennas were manufactured by means of a Computer Numerical Control (CNC) milling machine. The DH antenna was not considered because, as we found in the previous analysis, it has the worst polarization purity of all the antennas. Additionally, it is an intermediate step between both the D and DHG designs and therefore it is less interesting from a practical point of view. Photographs of the fabricated prototypes are shown in Figs. 5(a) and (b) for the D and DHG antennas, respectively. The feeding system is the same for both antennas, see details in Figs 5(c) and (d).

The measurements were performed in an anechoic chamber. A PNA network analyzer E8361C (Agilent Technologies) was used in the frequency range from 59 to $70 \mathrm{GHz}$ and the frequency span was discretized with steps of $50 \mathrm{MHz}$. The radiation patterns were measured by placing a transmitting horn antenna with linear polarization (Mi-Wave 261) at a distance of $1500 \mathrm{~mm}$ (the farfield distance at the operation frequency is at $720 \mathrm{~mm}$ ) from the antennas under test (AUTs). In each of these positions, the AUT was swept in elevation from $-90^{\circ}$ to $90^{\circ}$ with a step of $0.5^{\circ}$. As the test antenna (Mi-Wave 261) has linear polarization, in each measurement it was rotated in plane at orthogonal positions and from the recorded curves, the CP characteristics of the AUT were obtained.

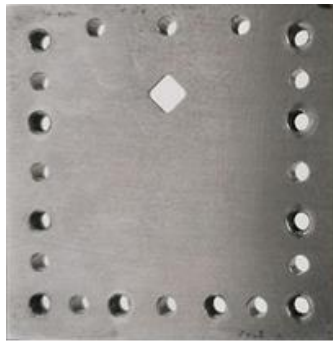

(a)

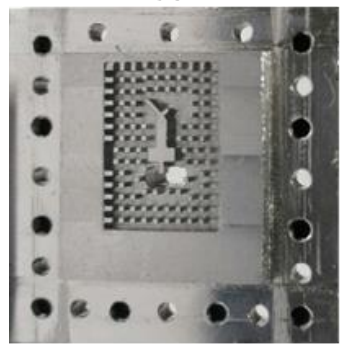

(c)

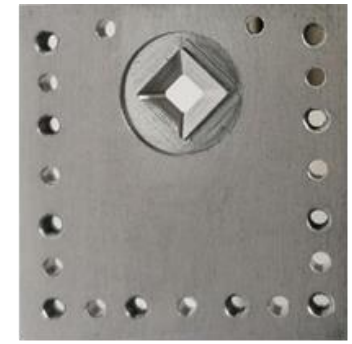

(b)

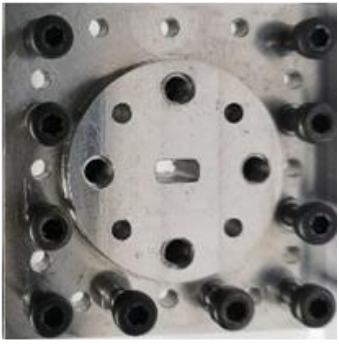

(d)
Fig. 5. Photographs showing a view of: (a) D antenna; (b) DHG antenna; (c) feeding system; (d) bottom WR-15 waveguide flange.

The reflection coefficient magnitude of both antennas is shown in Fig. 6(a), dotted curves. Both are below $-10 \mathrm{~dB}$ from 60 to $69.2 \mathrm{GHz}$ (note also that most of the band is below $-15 \mathrm{~dB}$ ), achieving an approximate $\mathrm{BW}$ of $9 \mathrm{GHz}$, which represents $14.24 \%$ of the center frequency, in good agreement with the simulation results. 

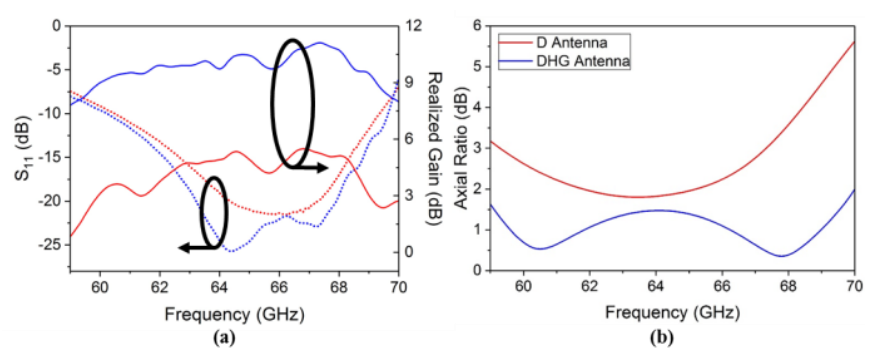

Fig. 6. Experimental results of the manufactured D (red) and DHG (blue) antennas. (a) Reflection coefficient and realized gain. (b) Axial ratio.

The characterization of the broadband gain was made by applying the gain transfer (or gain comparison) method [24][25]. First, two identical standard horn antennas (Mi-Wave 261) of known gain were carefully aligned and the received power within the frequencies of interest was recorded. Then, one of the standard horn antennas was replaced by the AUT. In this way, the gain is easily obtained, as shown in the solid lines of Fig. 6(a). The DHG antenna reaches a maximum of $11.12 \mathrm{~dB}$ at $67.3 \mathrm{GHz}$, whereas the D antenna has maximum of $5.49 \mathrm{~dB}$ at $66.8 \mathrm{GHz}$. Compared to simulations, in both cases there is a small improvement in the results achieved.

Fig. 6(b) shows the experimental AR [25]. Applying the same criterion of $A R<3 \mathrm{~dB}$, we find that the $\mathrm{D}$ antenna (red curve) has a $\mathrm{CP} \mathrm{BW}$ of $8 \mathrm{GHz}$ (from 59.9 to $67.2 \mathrm{GHz}$ ) or, equivalently, a fractional $\mathrm{BW}$ of $11.49 \%$. In the case of the DHG antenna (blue curve), the CP BW extends beyond the considered frequency range (note that the AR is below $2 \mathrm{~dB}$ in the plot) but the antenna is matched only from 60 to $69.2 \mathrm{GHz}$ as mentioned above. Thus, the practical operation $\mathrm{BW}$ is restricted by matching and reaches a value of $14.48 \%$. These results are in good agreement with the numerical calculations presented in the previous section.
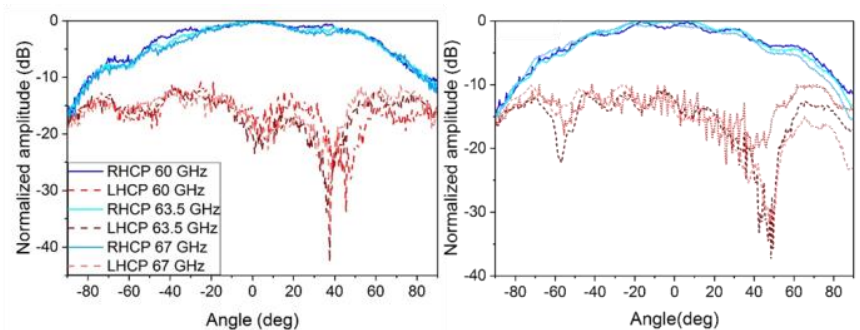

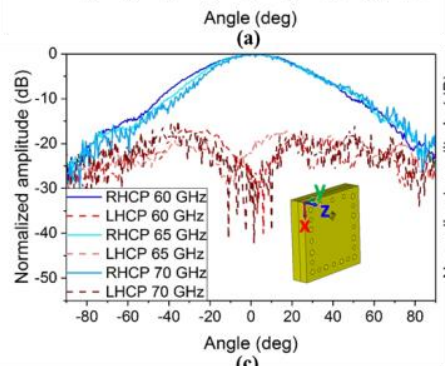

(c)

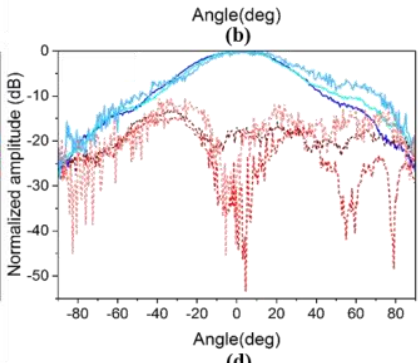

(d)
Fig. 7. Co-Polarized and Cross-Polarized measured radiation patterns at several selected frequencies. D Antenna (a) $\varphi=0^{\circ}, y-z$ plane (b) $\varphi=90^{\circ}, x-z$ plane. DHG Antenna (c) $\varphi=0^{\circ}, y-z$ plane (d) $\varphi=90^{\circ}, x-z$ plane. In the inset of panel (c) is a scheme of the antenna with the coordinate axes. The angle swept in all cases is the elevation, $\theta$.

The experimental radiation patterns of both antennas are shown in Fig. 7. In both cases, a sweep of $-90^{\circ} \leq \theta \leq 90^{\circ}$ was performed with a step of $0.5^{\circ}$, varying the frequency from 55 to
$70 \mathrm{GHz}$ with a step of $50 \mathrm{MHz}$. From all the evaluated frequency points, several representative frequencies were selected and represented in Fig. 7. The D antenna presents more than $15 \mathrm{~dB}$ of cross polarization isolation in both planes, as shown in Fig. 7(a) and Fig. 7(b). This parameter is even larger for the DHG antenna, which reaches values of more than $20 \mathrm{~dB}$ of cross polarization isolation; see Fig. 7(c) and Fig. 7(d). In addition, this last variant shows a clearer main lobe pointing at broadside in the radiation diagram, accounting for a larger directivity with respect to the basic D antenna, confirming the simulation results. In addition, the polarization rejection has an analogous trend in both cases, maintaining similar values and corroborating that the antenna polarization is RHCP.

Finally, Table II presents a comparison of our antennas with similar antennas reported in the literature. Structural characteristics and results achieved by each design are analyzed quantitatively. Certainly, most of the antennas mentioned here have greater gain than ours, so their bandwidth is restricted. Compared with previous designs, the antennas here devised have a better impedance and CP BW. Our designs have a unique radiant element and hence they are compact and simple, in contrast with other approaches based on arrays. Although the gain is below some of the designs found in the literature, their small dimensions and high BW make the D and DHG antennas strong candidates to be used in the 60 to $70 \mathrm{GHz}$ band.

TABLE II

COMPARISON BETWEEN DIFFERENT GW ANTENNAS

\begin{tabular}{cccccc}
\hline \hline & $\begin{array}{c}\text { Size } \\
(\mathrm{mm})\end{array}$ & $\begin{array}{c}\text { Number } \\
\text { of } \\
\text { elements }\end{array}$ & $\begin{array}{c}\text { BW } \\
(\mathrm{GHz})^{*}\end{array}$ & $\begin{array}{c}\text { Gain } \\
\text { Max } \\
(d B)\end{array}$ & $\begin{array}{c}\text { BW } \\
(\mathrm{GHz})^{+}\end{array}$ \\
\hline $\begin{array}{c}\text { D } \\
\text { Antenna } \\
\text { DHG }\end{array}$ & $30 \times 30$ & $1 \times 1$ & $60.5-69.3$ & 5.49 & $11.49 \%$ \\
Antenna & $30 \times 30$ & $1 \times 1$ & $60.3-69.6$ & 11.12 & $17.32 \%$ \\
Ref [14] & - & $1 \times 4$ & $74-83$ & 14.9 & $5.23 \%$ \\
& & & & & \\
Ref [16] & $53 \times 53$ & $4 \times 4$ & $29.5-31$ & 24.6 & $6.87 \%$ \\
Ref [17] & $7 \times 8.8$ & $1 \times 1$ & $72.1-82.8$ & 4 & $6 \%$
\end{tabular}

"Defined as $S_{11}<-10 \mathrm{~dB}$

${ }^{+}$Defined as $\mathrm{AR}<3 \mathrm{~dB}$

\section{CONCLUSION}

To sum up, in this paper we have designed several antennas with CP operating in the V-band of millimeter waves based on RGW technology. Two of these, D antenna and DHG antenna, were manufactured and measured. We have demonstrated that the use of two arms of different lengths in the feeding system, allows the generation of CP in a simple way, without the need of implementing and array along with a complex feeding network. The resulting antennas are small and very compact with excellent radiation characteristics. The simulated results and the measurements of the manufactured antennas are in good agreement. A broad operation BW of $14.48 \%(60.3-69.6 \mathrm{GHz})$ with an $\mathrm{AR}<3 \mathrm{~dB}$ is obtained by DHG antenna. It achieves a 
maximum gain of $11.12 \mathrm{~dB}$, a high value considering its small footprint $(30 \mathrm{~mm} \times 30 \mathrm{~mm})$ and simplicity. Both manufactured antennas manufactured have RHCP with more than $15 \mathrm{~dB}$ of cross polarization isolation. In comparison with similar antennas, our designs are competitive variants that can have different uses, either in the imminent implementation of the $5 \mathrm{G}$ or in any application that is supported in the $60-70 \mathrm{GHz}$ band. Furthermore, thanks to its fully metallic structure, they can be easily scaled to operate at other frequency bands such as terahertz.

\section{REFERENCES}

[11] J. Hirokawa and M. Zhang, Handbook of Antenna Technologies. Singapore: Springer Singapore, 2014.

[12] P.-S. Kildal, "Three metamaterial-based gap waveguides between parallel metal plates for $\mathrm{mm} / \mathrm{submm}$ waves," $3 r d$ Eur. Conf. Antennas Propag. (EuCAP 2009), vol. 8, no. March, pp. 28-32, 2009.

[13] T. Manabe et al., "Polarization dependence of multipath propagation and high-speed transmission characteristics of indoor millimeter-wave channel at $60 \mathrm{GHz}$," IEEE Trans. Veh. Technol., vol. 44, no. 2, pp. 268-274, May 1995.

[14] J. Xi, B. Cao, H. Wang, and Y. Huang, "A novel $77 \mathrm{GHz}$ circular polarization slot antenna using ridge gap waveguide technology," in 2015 Asia-Pacific Microwave Conference (APMC), 2015, pp. 1-3.

[15] T. Li and F. Fan, "Design of ka-band $2 \times 2$ circular polarization slot antenna array fed by ridge gap waveguide," in 2017 Sixth AsiaPacific Conference on Antennas and Propagation (APCAP), 2017, pp. 1-3.
[16] M. Ferrando-Rocher, J. I. Herranz-Herruzo, A. Valero-Nogueira, and A. Vila-Jimenez, "Single-Layer Circularly-Polarized Ka-Band Antenna Using Gap Waveguide Technology," IEEE Trans. Antennas Propag., vol. 66, no. 8, pp. 3837-3845, Aug. 2018.

[17] F. Fan and Z. Yan, "High gain circularly polarized slot antenna based on microstrip-ridge gap waveguide technology," in 2017 11th European Conference on Antennas and Propagation (EUCAP), 2017, pp. 1669-1672.

[18] A. Torres-Garcia, F. Marante, A. Tazon, J. Vassal'lo, J. Teniente, and M. Beruete, "Broadband circular polarized field generation in single layer microstrip patch antennas," in 2016 10th European Conference on Antennas and Propagation (EuCAP), 2016, pp. 1-4. A. Vosoogh, A. U. Zaman, and J. Yang, "Simple and Broadband Transition Between Rectangular Waveguide and Groove Gap Waveguide for MM-Wave Applications," in 2018 IEEE International Symposium on Antennas and Propagation \& USNC/URSI National Radio Science Meeting, 2018, pp. 1101-1102.

[20] A. A. Brazalez, E. Rajo-Iglesias, J. L. Vazquez-Roy, A. Vosoogh, and P.-S. Kildal, "Design and Validation of Microstrip Gap Waveguides and Their Transitions to Rectangular Waveguide, for Millimeter-Wave Applications," IEEE Trans. Microw. Theory Tech., vol. 63, no. 12, pp. 4035-4050, Dec. 2015.

[21] B. Molaei and A. Khaleghi, "A Novel Wideband Microstrip Line to Ridge Gap Waveguide Transition Using Defected Ground Slot," IEEE Microw. Wirel. Components Lett., vol. 25, no. 2, pp. 91-93, Feb. 2015.

[22] M. Al Sharkawy and A. A. Kishk, "Design of waveguide to ridge gap waveguide transition using probe excitation," in The 8th European Conference on Antennas and Propagation (EuCAP 2014), 2014, no. EuCAP, pp. 946-949.

[23] A. A. Brazalez, A. U. Zaman, and P.-S. Kildal, "Design of a coplanar waveguide-to-ridge gap waveguide transition via capacitive coupling," in 2012 6th European Conference on Antennas and Propagation (EUCAP), 2012, pp. 3524-3528.

[24] G. A. Hurd, "IEEE Standard Test Procedures for Antennas," Electron. Power, vol. 26, no. 9, p. 749, 1980

[25] Bee Yen Toh, R. Cahill, and V. F. Fusco, "Understanding and measuring circular polarization," IEEE Trans. Educ., vol. 46, no. 3, pp. 313-318, Aug. 2003.

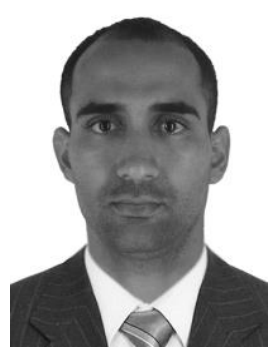

Dayan Pérez-Quintana was born in Cuba in 1991. He received the Degree in Telecommunication Engineering from the Instituto Superior Politécnico José Antonio Echeverría (ISPJAE), Havana, Cuba, in 2015. He is currently working as a Predoctoral Research Fellow in the Antenna Group in the Electrical, Electronic and Communication Engineering Department of the Public University of Navarre (UPNA), Spain. His interests include millimeter wave and $\mathrm{THz}$ components and antennas and metamaterials

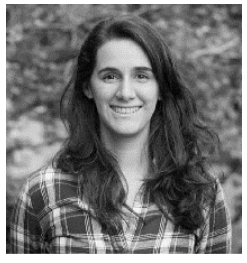

Alicia E. Torres-García was born in Cuba in 1990. She received the Degree in Telecommunication Engineering from the Instituto Superior Politécnico José Antonio Echeverría (ISPJAE), Havana, Cuba, in 2013. She is currently working as a Predoctoral Research Fellow in the Antenna Group in the Electrical, Electronic and Communication Engineering Department of the Public University of Navarre (UPNA), Spain. Her interests include millimeter wave and $\mathrm{THz}$ components and antennas, metamaterials, planar antennas and different research fields in the microwave range. 


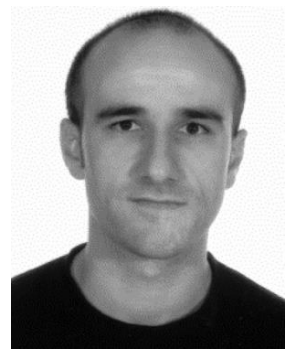

Iñigo Ederra (M'15) received the Ingeniero de Telecomunicación and Ph.D. degrees from the Public University of Navarre (UPNA), Pamplona, Spain, in 1996 and 2004, respectively.,In 1997, he joined the Microwave and Millimetre Wave Group, UPNA. From 1999 to 2000, he was with the European Space Research and Technology Centre, European Space Agency, Noordwijk, The Netherlands, where he was involved in electromagnetic bandgap materials and their applications in the field of antennas. Since 2001, he has been with the Antenna Group, UPNA. In 2002, he joined the Rutherford Appleton Laboratory, Chilton, Didcot, U.K., as Visitor Scientist, participating in ESA's Startiger I Project. He is currently an Associate Professor with UPNA and is a member of the Institute of Smart Cities. His research interests include electromagnetic bandgap materials, metamaterials, and metasurfaces and their applications in microwave, millimeterwave, and $\mathrm{THz}$ components and antennas.

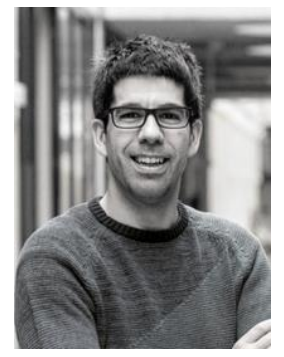

Miguel Beruete was born in Pamplona, Spain, in 1978. He received the M.Sc. and Ph.D. degrees in telecommunication engineering from the Public University of Navarre (UPNA), Pamplona, in 2002 and 2006, respectively.

From 2002 to 2007, he was a PreDoctoral Researcher, under FPI fellowship, with the Electrical and Electronic Engineering Department, UPNA. In 2005, he was a Visiting Researcher with the University of Seville, Seville, Spain, as a part of his Ph.D. research. From 2007 to 2009, he was with the Electronics Department, Technological Center CEMITEC, Noáin, Spain, where he was involved in the development of high frequency communication devices. In 2009, he joined TERALAB, UPNA, as a Post-Doctoral Researcher on the Consolider EMET Engineering Metamaterials Project. From 2012 to 2016, he was a Ramon y Cajal Fellow Researcher with UPNA, where he became an UPNA Distinguished Researcher. Since 2017, he has been an Associate Professor with the Antennas Group-TERALAB, UPNA, where he supervises several Ph.D. and M.Sc. theses and leads the TERALAB Laboratory. He has authored more than 130 indexed articles, 5 book chapters, more than 300 conference communications (several invited). He holds three patents. His current research interests include terahertz sensing and communication technology, including metamaterials, plasmonics, extraordinary transmission structures, leaky-wave antennas, nanoantennas, and in general quasi-optical devices.

Dr. Beruete was a recipient of the Ph.D. Prize from UPNA for the Best Doctoral Thesis from 2006 to 2007; Research Prize UPNA for the best scientific contribution made during 2017 and 2018 in the Areas of Exact, Biological, Medical and Technological Sciences; three CST University Publication Awards for the best international journal publication using CST in 2005, 2012, and 2016; the XII Talgo Award of Technological Innovation in 2011; and several awards of international conferences. He is a reviewer for more than 50 international journals. 